\title{
One-Dimensional Staged Self-Assembly
}

\author{
Erik D. Demaine ${ }^{1}$, Sarah Eisenstat ${ }^{1}$, \\ Mashhood Ishaque ${ }^{2}$, and Andrew Winslow ${ }^{2}$ \\ 1 MIT Computer Science and Artificial Intelligence Laboratory, \\ Cambridge, MA 02139, USA, \{edemaine, seisenst\}@mit.edu \\ 2 Department of Computer Science, Tufts University, \\ Medford, MA 02155, USA, \{mishaque, awinslow\}@cs.tufts.edu
}

\begin{abstract}
We introduce the problem of staged self-assembly of onedimensional nanostructures, which becomes interesting when the elements are labeled (e.g., representing functional units that must be placed at specific locations). In a restricted model in which each operation has a single terminal assembly, we prove that assembling a given string of labels with the fewest stages is equivalent, up to constant factors, to compressing the string to be uniquely derived from the smallest possible context-free grammar (a well-studied $O(\log n)$-approximable problem). Without this restriction, we show that the optimal assembly can be substantially smaller than the optimal context-free grammar, by a factor of $\Omega(\sqrt{n / \log n})$ even for binary strings of length $n$. Fortunately, we can bound this separation in model power by a quadratic function in the number of distinct glues or tiles allowed in the assembly, which is typically small in practice.
\end{abstract}

Keywords: context-free grammar, Wang tile, DNA computing, complexity

\section{Introduction}

Self-assembly is the study of how small particles (typically at the nanoscale, where electrostatic forces overwhelm gravity) interact with each other to conglomerate into larger objects. In theoretical computer science, the standard model is the tile assembly model [10] in which the system begins with infinitely many copies of certain square tiles, each with specified glues on the four sides, and tiles translate nondeterministically in the plane until they attach to each other at matching glues. This model effectively enables performing computation, but out of simple geometric parts, and at the cost of physical space resulting from the assembly.

The most studied problem in the tile assembly model is to determine the number of distinct tile types required to assemble a given shape (made out of unit squares). An obvious upper bound is the area of the shape, but in many cases fewer tiles suffice, by building computation into the construction. For example, an $n \times n$ square requires $\Theta(\log n / \log \log n)$ distinct tile types (and glues), by embedding a base- $\log n$ counter, while an $n \times 1$ rectangle requires $\Theta(n)$ tile 
types. The most general result is that any shape, scaled by a sufficiently large factor, can be constructed from $O(K / \log K)$ tile types (and glues), where $K$ is the Kolmogorov complexity of the shape [9]. Unfortunately, the scale factor is polynomial in the running time of the Turing machine generating the shape, which is at least the area of the shape. So this result does not directly address the tile complexity of a specific shape, though it suggests that it is difficult to characterize.

An alternate approach is offered by staged self-assembly 2] in which the system's tile set can change in a sequence of stages, in particular by an experimenter mixing two systems together. In this model, it is possible to make any shape using a constant number of tile types (and glues); as a result, the main objectives are to minimize both the number of mix operations (work for the experimenter) and the number of stages that must be executed sequentially (makespan or wait time). For example, both an $n \times n$ square and an $n \times 1$ rectangle can be assembled using $O(1)$ tiles and glues and $O(\log n)$ mixes and stages. Again we lack a general characterization of the number of mixes and stages required for a desired 2D shape.

Our personal communication with bioengineers suggests that the staged assembly model is natural and practical, essentially exposing the experimenter's ability to perform actions as part of a computation/assembly. Furthermore, the results are more practical, as it is difficult in practice to design many different glues that attract only in pairs, without any attraction between unpaired glues. Assembling a $1000 \times 1$ rectangle would be impractical without staging (requiring 1000 tile types and 999 distinct glues), but is extremely practical with staging (requiring only 6 tile types, 3 distinct glues and 10 stages).

In this paper, we aim to characterize the resources required to staged-assemble a one-dimensional object. Just making a $1 \times n$ rectangular shape is trivial, so this direction has so far been overlooked. But in practice, experimenters often want to build an object that not only takes on a desired shape but also carries out a desired functionality. A typical example is to arrange nanodots or bioagents in a particular pattern within a shape ${ }^{3}$ We model this problem as constructing a labeled shape, where each unit square has a label within a fixed alphabet, and each tile type used to build the shape also has a label, which must match in construction. Thus the input to the problem is a string of labels, and the goal is to find a staged assembly with few glues, mixes, and stages. In fact we show that four glues and $O(\log n)$ stages always suffice, so a single objective remains: minimize the mixes.

The problem of computing a minimal tile assembly system that produces a labeled shape has been studied previously. Heuristic approaches have been developed to find the smallest tile set that uniquely assembles an input labeled shape [6], and the problem of finding a minimum-size tile set has been shown to be NP-hard [6].

We successfully characterize the number of mixes required to staged-assemble a string in two natural situations. In the first setting (Section 4), we restrict

\footnotetext{
${ }^{3}$ Personal communication with Hyunmin Yi, 2008-2010.
} 
mixing operations to produce a single terminal assembly for use in the next mix. This restriction seems to be common to all previous staged-assembly algorithms [2], but we do not know it to be practically motivated. In the second setting (Section 5.5), we allow multiple "parallel assemblies" resulting from a mix (a new though natural idea), and consider the more natural restriction that the number of glues is constant. In either setting, we show that the minimum number of mixes is within a constant factor of the smallest context-free grammar that generates exactly the given string. The latter problem is well-studied, has a polynomialtime $O(\log n)$-approximation algorithm based on Lempel-Ziv compression, and likely has no $o(\log \log n)$-approximation [1/5/7/8].

We show that our relations are nearly tight by constructing a family of strings (Section 5) showing a separation in power between (unrestricted) staged assembly and context-free grammars. Specifically, an $n$-bit binary string can be assembled using $O(k)$ glues with $O(k)$ mixes but requires a context-free grammar of size $\Omega\left(k^{2}\right)$, for a ratio of $\Omega(k)$. Our upper bound shows that the worst-case separation is $O\left(k^{2}\right)$. As a function of $n$ (with an unbounded number of glues), we prove that the ratio is $\Omega(\sqrt{n / \log n})$. In practice, small feature sizes make the number of glues typically small, in which case context-free grammars are actually a good approximation to optimal staged self-assemblies.

The labeled 1D staged self-assembly model offers a balance of tractability, being easier than general staged assembly by reducing the dimension to 1 , yet harder (and more practical) by adding labels to the target shape. The connections we show to context-free-grammar compression illustrate that the problem is difficult, yet for the case of many glues, still not fully understood. The approximation algorithm resulting from our study is simple and efficient, having been implemented in an online web system 4 which is currently being considered for practical use by the Tufts Department of Bioengineering, in a setting where labeled 1D assemblies are of significant interest.

\section{Context-Free Grammars}

Definition 1. A context-free grammar $(C F G)$ is defined as a 4-tuple $(\Sigma, \Gamma, S, \Delta)$ where $\Sigma$ is a set of terminal symbols, $\Gamma$ is a set of non-terminal symbols, $S$ is a special element of $\Gamma$ called the start symbol and $\Delta$ is a set of productions.

Each production consists of a left-hand side containing a single non-terminal symbol, and a right-hand side containing a (non-empty) sequence of terminal and non-terminal symbols. A CFG derives a string by repeated replacement of nonterminal symbols with strings of terminal and non-terminal symbols according to the productions in $\Delta$, beginning with the single symbol $S$. The language of a CFG is the set of derivable strings consisting solely of terminal symbols.

Definition 2. The size of a context-free grammar $G$, denoted $|G|$, is the total number of symbols appearing on the right-hand sides of the productions in $G$.

\footnotetext{
4 http://selfdisassembler.appspot.com/
} 
Note that this definition counts the total number of symbols, so symbols appearing multiple times contribute to the count multiple times. In this paper we consider only CFGs that are deterministic (with only one production per left-hand side) and generate exactly one string.

Definition 3. A restricted context-free grammar (written RCFG) is a $C F G$ which is deterministic and has a language consisting of a single string.

For an RCFG $G$ deriving a string $s$, the parse tree of $G$ is the tree created by beginning with a single node with label $S$ (the start symbol), and adding children to a leaf node for each production applied. The result is a tree where each internal node is a non-terminal symbol, each leaf node is a terminal symbol, and an in-order traversal of the leaves gives the string $s$.

Definition 4. The smallest grammar problem is the following: given an input string $s$, find the smallest RCFG deriving $s$.

Any RCFG $G$ has exactly one parse tree. Each internal node in the parse tree has a corresponding non-terminal symbol from $G$. If we merge all such nodes with the same non-terminal, the result is a directed acyclic graph (DAG) called the parse DAG. See Figure 1 for an example of an RCFG and its parse tree and parse DAG.

$$
\begin{aligned}
& A \rightarrow B C \\
& B \rightarrow D C \\
& C \rightarrow a a \\
& D \rightarrow c c
\end{aligned}
$$
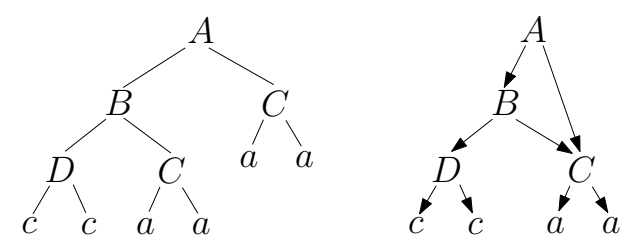

Fig. 1. A restricted context-free grammar (RCFG) and its corresponding parse tree and parse DAG.

\section{$3 \quad$ Staged Self-assembly}

In this section we describe the 1D labeled staged self-assembly system model. The model described here is a variant of the staged self-assembly model defined in 2. In this model, individual building blocks are 2D square-shaped tiles that translate in the plane. Each tile has four sides (north, south, east, and west) and has glues on its east and west sides. Each tile also has a label $(a, b, c, \ldots)$. We denote a tile $x_{1}\left[x_{2}\right] x_{3}$ where $x_{1}$ is the west glue, $x_{2}$ is the label, $x_{3}$ is the east glue (e.g., 1[a]2).

Tiles combine when the pair of glues on the west side of one tile and east side of the other are complementary. We denote glue values by numbers (e.g., 
$1,2, \ldots)$, and the complementary glues are denoted $1^{\prime}, 2^{\prime}, \ldots$ In this paper we use the convention that east glues are always complementary (prime) glues. So a tile $1[a] 2$ actually has east glue $2^{\prime}$.

When tiles combine they create assemblies (and we consider tiles to be a special case of assemblies). The labels of each individual tile combine to form a label string of the assembly consisting of the labels of the combined tiles in order. For example, $1[a] 2$ and $2[b] 3$ combine to form the assembly $1[a b] 3$. Note that the east glue of $1[a] 2$ and west glue of $2[b] 3$ have disappeared: they are on the interior of the assembly and are omitted for clarity. Assemblies can also be combined to form larger assemblies. The size of an assembly is the number of tiles it contains.

Initially each tile type exists in a separate bin. When bins are mixed, the assemblies present in each bin are free to attach to each other. The products of each mixing are terminal assemblies: assemblies that do not attach to any other assemblies. All other assemblies produced during the mixing are assumed to be filtered out before the bin is combined with other bins.

A self-assembly system instance is defined by the starting tiles and a mix DAG defining bins and the orders in which they are mixed. The mix DAG is a rooted DAG: a DAG with only one node (the root) without in-edges. where each node represents a bin and the edges leaving it point to the bins whose contents are mixed into this bin. Each leaf of the DAG is a bin of a single tile type.

Definition 5. A self-assembly system (SAS) is a one-dimensional labeled staged self-assembly instance that produces a single goal assembly and is defined by a mix DAG and a unique tile type for each leaf of the DAG.

Definition 6. The size of a $S A S A$ (denoted $|A|$ ) is the number of edges in its mix DAG.

The goal assembly produced by a SAS must appear in the bin corresponding to the root node of the mix DAG. Reading the labels of an assembly from west to east defines a string which we call the label string of the assembly. The label string of the goal assembly is the string generated by the SAS.

In previous staged-assembly constructions [2], each bin has a single assembly produced in it by mixing the contents of two other bins (which also contain single items). However the model as defined does not require that each bin contains a single assembly. A mix DAG in which one or more bins has multiple assemblies is said to use bin parallelism. We distinguish a self-assembly system instance that does not use bin parallelism as a single self-assembly system (SSAS).

Definition 7. A single self-assembly system (SSAS) is a SAS in which no bin contains more than one distinct assembly.

Definition 8. The minimum SSAS problem is the following: given an input string $s$, find a smallest SSAS generating an assembly with label string $s$. 


\section{Equivalence between RCFGs and SSASs}

In this section we show that converting between an RCFG $G$ deriving a string $s$ and a SSAS instance $A$ assembling a labeled assembly with label $s$ is possible with only a constant-factor scaling. As a result, any algorithm generating an $O(f(n))$ approximation to either the minimum grammar problem or the minimum SSAS problem implies an $O(f(n))$-approximation algorithm for the other.

\subsection{Converting RCFGs to SSASs}

Let $G$ be an RCFG deriving a string $s$. We begin by converting $G$ to an equivalent RCFG $G^{\prime}$ with at most two symbols on the right-hand side of each production (such a CFG is called a binary $C F G$ ).

Recall that each rule is represented by a subtree of the parse DAG consisting of a root node (the left-hand side symbol) and its children (the right-hand side symbols). This subtree can obviously be converted into a binary tree which has size at most twice the number of leaves, and is at most twice the size of the original subtree. So each rule can be expanded to a set of binary rules with at most twice as many symbols. As a result, $G$ is at most doubled in size and thus $\left|G^{\prime}\right| \leq 2|G|$.

Next we convert each production of $G^{\prime}$ to a SSAS mixing. However, a problem occurs if the same non-terminal appears as a right-hand side symbol in several production rules. Recall that a production in the grammar specifies the left-toright order in which the right-hand side symbols appear, while the west-to-east order in which assemblies attach is determined by their glues. To produce exactly the assembly desired in a mixing requires combining its subassemblies with the correct glues.

To resolve this issue, we construct several copies of every assembly: one for each possible west/east glue pair. Since the grammar is binary, at most two assemblies are mixed in each bin and so three glue pairs is enough to uniquely specify the mixing product. Given a production $A \rightarrow B C$, we create six bins and six mixings that assemble the six west/east glue pair combinations for $A$ from the six west/east glue pair combinations for $B$ and $C$ (see Table 1 ).

Table 1. The set of mixings to produce all necessary glue pair variations for assembly $A$ in the production $A \rightarrow B C$.

\begin{tabular}{|l|l|l|l|l|l|l|}
\hline A glues & $(1,2)$ & $(1,3)$ & $(2,1)$ & $(2,3)$ & $(3,1)$ & $(3,2)$ \\
B glues & $(1,3)$ & $(1,2)$ & $(2,3)$ & $(2,1)$ & $(3,2)$ & $(3,1)$ \\
C glues & $(3,2)$ & $(2,3)$ & $(3,1)$ & $(1,3)$ & $(2,1)$ & $(1,2)$ \\
\hline
\end{tabular}

Lemma 1. A parse DAG for a binary $R C F G G^{\prime}$ deriving string s can be converted to a valid SSAS A of size at most $6\left|G^{\prime}\right|$ that constructs an assembly with label string $s$. 
Proof. We build the mix DAG of $A$ in the following way: For each symbol (terminal and non-terminal) create 6 bins for the glue-pair variants of the symbol. For each production $A \rightarrow B C$ of $G^{\prime}$, mix the 6 bins of $B$ and $C$ into the 6 bins of $A$ as in Table 1. The resulting mix DAG has 6 bins for each symbol of $G^{\prime}$, each containing a unique glue-pair variant of an assembly with label string corresponding to the string derived by the symbol in $G^{\prime}$. Each edge of the parse DAG of $G^{\prime}$ is converted to 6 edges in the mix DAG of $A$, one for each glue-pair variant. So $|A| \leq 6\left|G^{\prime}\right|$.

Theorem 1. Given an $R C F G G$ deriving a string s, the algorithm described in Section 4.1 computes a SSAS instance $A$ with $|A| \leq 12|G|$ that produces an assembly with label string $s$.

Proof. The algorithm converts $G$ to a binary RCFG $G^{\prime}$, and then converts $G^{\prime}$ to a mix DAG for $A$. By Lemma 1, $|A| \leq 6\left|G^{\prime}\right|$. So $|A| \leq 6\left|G^{\prime}\right| \leq 12|G|$.

\subsection{Converting SSASs to RCFGs}

Let $A$ be a SSAS constructing an assembly with label string $s$. We perform a (nearly) one-to-one mapping from the mix DAG of $A$ to the parse DAG of a grammar $G$. For each leaf bin of $A$, create a terminal symbol in $G$ equal to the label string of the tile in the bin. For each non-leaf bin of $A$, create a non-terminal symbol in $G$. For each mixing in $A$ combining the contents of bins $b_{1}, b_{2}, \ldots, b_{k}$ into bin $B$, create a production in $G$ with $B$ on the left-hand side and $b_{1}$ through $b_{k}$ on the right-hand side in the order they combine when mixed in $B$.

Theorem 2. For any SSAS A constructing an assembly with label string s, an $R C F G G$ deriving $s$ can be constructed from $A$ such that $|G|=|A|$.

Proof. The terminal symbols of $G$ are equal to the label strings of their corresponding tiles. Each mixing in $A$ produces a single assembly with a label string equal to the string derived by the corresponding non-terminal symbol in $G$, because the production orders the right-hand side symbols in the same order that they combine in $A$. So the start symbol of $G$ derives a string equal to the label string of the assembly produces in the root of the mix DAG of $A$. So $G$ derives $s$. Each edge of the mix DAG of $A$ causes a right-hand side symbol to appear in a production of $G$. So $|G|=|A|$.

\subsection{Approximation Equivalence}

The conversions presented above in Sections 4.1 and 4.2 immediately imply that approximation algorithms for either problem transfer to the other, at a constantfactor loss.

Corollary 1. An $O(f(n))$-approximation algorithm for the smallest grammar problem exists if and only if an $O(f(n))$-approximation algorithm for the minimum SSAS problem exists. 
In practice this theorem makes computing efficient SSAS instances easier, as several $O(\log n)$-approximation algorithms to the minimum grammar problem exist $17 / 8$. We have taken advantage of this fact to produce a software tool for finding $O(\log n)$ approximations to the minimum SSAS problem in $O(n)$ time using the algorithm by Sakamoto [8].

This result also suggests that finding an improved approximation algorithm for the minimum SSAS problem is unlikely. In 2002, Lehman showed that a polynomial-time approximation algorithm for the smallest grammar problem with factor $o\left(\frac{\log n}{\log \log n}\right)$ would enable progress on "a difficult algebraic problem in a well-studied area" [5].

\section{Separation Between SASs and RCFGs}

Now we show that the general 1D staged self-assembly model (SAS) is not equivalent to RCFGs. The proof is constructive: we give a set of strings and describe a set of SAS instances that produce assemblies with these label strings. We then show that any CFG producing these strings is asymptotically larger than the SAS instance producing the corresponding label string.

It might appear obvious that allowing bin parallelism should allow a reduction in the amount of work needed to construct an assembly. However, using parallelism has two costs that make saving work difficult. First, for any minimal SAS, no two assemblies in the same bin may share a common glue (this is proven in Lemma ??). As a result, additional parallelism requires more unique glues, which in turn requires more starting bins, and thus more work. Second, since the goal of an assembly system is to construct a single goal assembly, bins with parallelism must eventually be "collapsed" into a single bin with a single object (otherwise the parallelism was extraneous). Collapsing bins with parallelism involves adding tiles to join the various assemblies together, and since the glues on each assembly are unique, creating and mixing the joining tiles requires additional work proportional to the amount of parallelism in the bin.

\subsection{A Set of Strings $S_{k}$}

To derive an asymptotic bound between SASs and RCFGs, we use a special set of strings that can be built by small SASs but require large RCFGs. Each string consists of a sequence of interleavings of pairs of smaller strings. We will consider only odd values of $k$ for the remainder of the paper.

Let $\operatorname{BinARY}(i, \ell)$ be the binary representation of $i$ of length $\ell$. The following is a function used to double every character in a string:

$$
\operatorname{Double}\left(b_{1} b_{2} b_{3} \ldots b_{n}\right)=b_{1} b_{1} b_{2} b_{2} b_{3} b_{3} \ldots b_{n} b_{n}
$$

We define $s_{1} \circ s_{2}$ to be the concatenation of string $s_{1}$ followed by $s_{2}$. We wish to encode a number of distinct "characters" in binary. To construct a suitably hard-to-compress string, we want to ensure that the beginning and end of each encoding are clearly delineated. To that end, we define the following strings for all values of $k$ and all values of $i<2 k$ : 
Definition 9. $A_{k, i}=(01) \circ \operatorname{Double}(\operatorname{BinaRy}(i, 1+\lceil\log k\rceil)) \circ(01)$.

Note that each such string has length $6+2\lceil\log k\rceil$.

We wish to use these characters to construct a string with a lot of structure (so that it is efficiently constructible using a SAS) but minimal repetition (so that it is not efficiently constructible using a CFG). To minimize repetition, we choose a string with the property that no sequential pair of characters is repeated. We define the following functions, which are permutations for $0 \leq x<k$ :

$$
\pi_{k, 0}(x)=2 x \bmod k \quad \pi_{k, 1}(x)=2 x+1 \bmod k
$$

We use these two simple functions to construct a more complex permutation.

Definition 10. Say that the bits of $\operatorname{BinARY}(i, \ell)$ are $b_{1}, \ldots, b_{\ell}$. Then

$$
\Pi_{k, \ell, i}(j)=\pi_{k, b_{\ell}}\left(\pi_{k, b_{\ell-1}}\left(\ldots \pi_{k, b_{2}}\left(\pi_{k, b_{1}}(j)\right) \ldots\right)\right) .
$$

Because $k$ is odd, this function is a permutation for $0 \leq j<k$. In addition, as long as $0 \leq i<2^{\ell}$, this function has the property that $\Pi_{k, \ell, i}(j)=\left(2^{\ell} \cdot j+i\right) \bmod k$. This means that for fixed values of $k, \ell$, and $j$, each value of $i$ such that $0 \leq i<k$ will generate a different value of $\Pi_{k, \ell, i}(j)$.

This permutation can be used to ensure that no sequential pair of characters is repeated. To do so, we construct pairs of characters as follows:

$$
C_{k, i, j}=A_{k, j} \circ(01)^{\lceil\log k\rceil} \circ A_{k, k+\Pi_{k,\lceil\log k\rceil, i}(j)} \circ(01)^{\lceil\log k\rceil} .
$$

We concenate these pairs to construct $P_{k, i}=C_{k, i, 0} \circ C_{k, i, 1} \circ \ldots \circ C_{k, i, k-1}$. Note that the length of each $C_{k, i, j}$ is $12+8\lceil\log k\rceil$, and therefore the length of each $P_{k, i}$ is $(12+8\lceil\log k\rceil) \cdot k$.

We concatenate each $P_{k, i}$ to get the string we wish to compress:

Definition 11. $S_{k}=01 \circ P_{k, 0} \circ 01 \circ P_{k, 1} \circ 01 \circ \ldots \circ 01 \circ P_{k, k-1} \circ 01$

In the next two subsections we give bounds on compressing $S_{k}$ using both a RCFG and a SAS.

\subsection{A SAS Upper Bound for $S_{k}$}

Now we describe a self-assembly system using bin parallelism that produces an assembly with $S_{k}$ as its label string. The system is broken down into several subsystems described in this section. A diagram of the SAS for $S_{3}$ is seen in Figure 2

Constructing $\boldsymbol{A}_{\boldsymbol{k}, \boldsymbol{i}}$ for all $\mathbf{0} \leq \boldsymbol{i}<\mathbf{2} \boldsymbol{k}$ Say that we are given $2 k$ glue pairs $x_{i}, y_{i}$, and that we want to assemble $x_{i}\left[A_{k, i}\right] y_{i}$. for each $0 \leq i<2 k$. In addition, say that we are given three additional glues $g_{0}, g_{1}$, and $g_{2}$ for use in our construction. Let $\ell=1+\lceil\log k\rceil$.

For each binary string $s$ of length $\leq \ell$, we construct two bins: $I_{s}$ and $F_{s}$. Let $s=t \circ b$, where $b \in\{0,1\} . I_{s}$ will contain an assembly with glue $g_{0}$ on the left, 


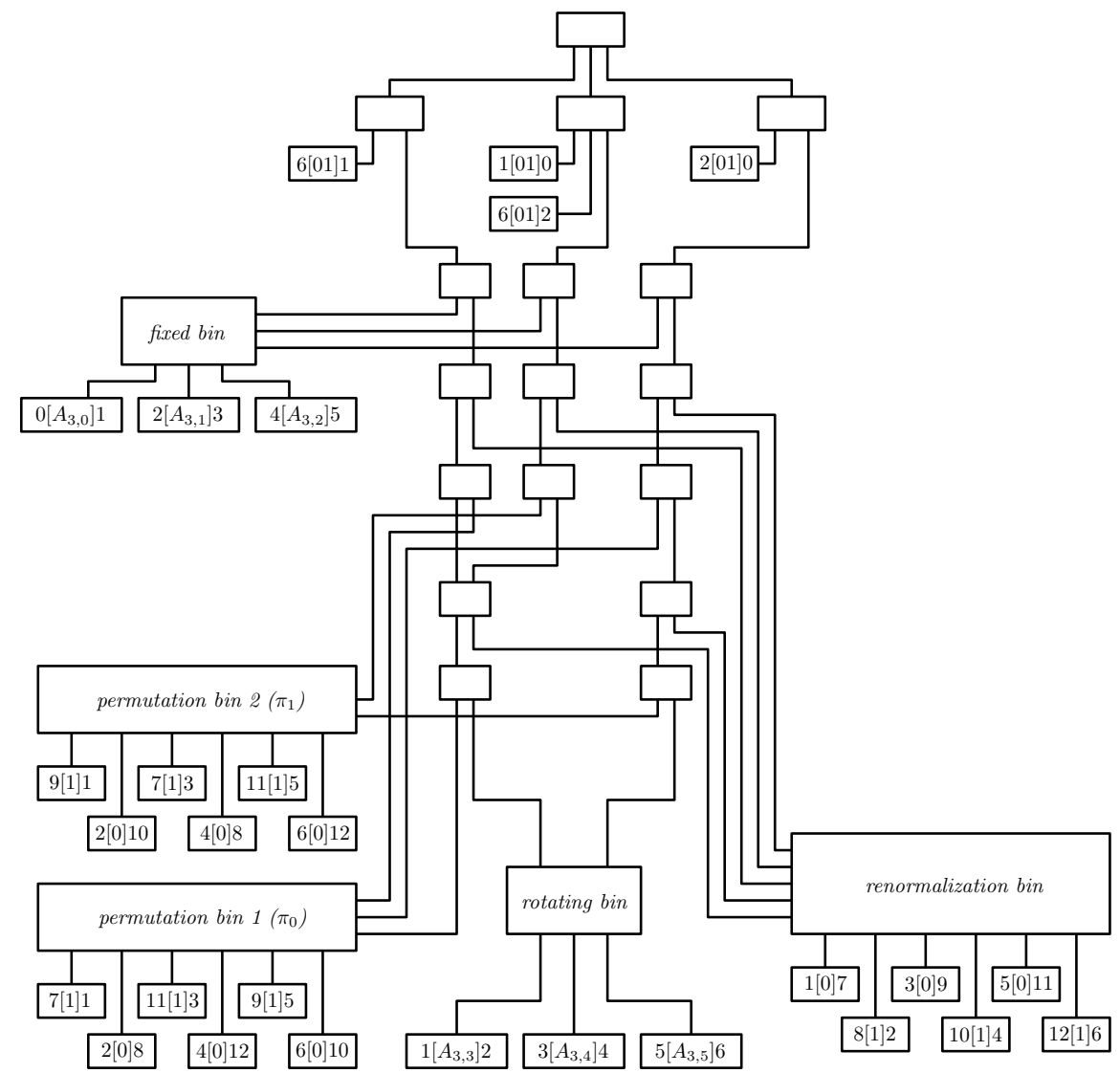

Fig. 2. The mix DAG for a SAS generating an assembly with label string $S_{3}$.

glue $g_{1}$ on the right, and the label Double $(t) \circ b . F_{s}$ will contain an assembly with glue $g_{0}$ on the left, glue $g_{2}$ on the right, and the label Double $(s)$. The assembly in bin $I_{s}$ will be constructed by adding the tile $g_{2}[b] g_{1}$ to the assembly in bin $F_{t}$. The assembly in bin $F_{s}$ will be constructed by adding the tile $g_{1}[b] g_{2}$ to the assembly in bin $I_{s}$.

To finish this construction, we add the constant-sized assemblies $x_{i}[01] g_{0}$ and $g_{2}[01] y_{i}$ to the bin $F_{\operatorname{Binary}(i, \ell)}$. This ensures that for $0 \leq i<2 k$, the bin $F_{\text {Binary }(i, \ell)}$ contains an assembly with the label $A_{k, i}$. The total number of bins required for this construction is $\Theta(k)$.

Fixed and Rotating Bins The fixed bin contains the following set of tiles:

$$
0\left[A_{k, 0}\right] 1,2\left[A_{k, 1}\right] 3, \ldots,(2 k-2)\left[A_{k, k-1}\right](2 k-1)
$$


The rotating bin contains the following set of tiles:

$$
1\left[A_{k, k+0}\right] 2,3\left[A_{k, k+1}\right] 4, \ldots,(2 k-1)\left[A_{k, k+(k-1)}\right](2 k)
$$

Permutation and Renormalization Bins Permuting the assemblies in the rotating bin is simulated by attaching permutation tiles to the east and west ends of those assemblies. The permutations $\pi_{k, 0}$ and $\pi_{k, 1}$ are implemented as two sets of $2 k$ tiles, each set in a separate permutation bin. A third set of $2 k$ tiles are put in a renormalization bin used to solve a technical issue with the permutation bins.

The permutation bin for $\pi_{k, 0}$ has tiles that replace the primal glues of assembly $i(2 i+1$ and $2 i+2)$ with the dual glues of assembly $\pi_{k, 0}(i)\left(2 \pi_{k, 0}(i)+\right.$ $1+(2 k+1)$ and $\left.2 \pi_{k, 0}(i)+2+(2 k+1)\right)$ for all assemblies $0 \leq i \leq k-1$. The permutation bin for $\pi_{k, 1}$ is constructed analogously. Each tile attaches to either the east or west end of the assembly and correspondingly has the primal and dual glues on its east and west sides. The tiles attaching to the east end of the assembly have the label 0 ; the tiles attaching to the west end of the assembly have the label 1.

The renormalization bin has a pair of tiles for changing the dual glues of assembly $i((2 i+1)+(2 k+1)$ and $(2 i+2)+(2 k+1))$ to its primal glues $((2 i+1)$ and $(2 i+2))$. The tiles attaching to the east end of each assembly have the label 1 ; the tiles attaching to the west end of each assembly have the label 0.

Creating Interleaved Assemblies The permutation and renormalization bins are applied in a branching manner to produce all permutation sequences of length $\ell$. First $\pi_{k, 0}$ and $\pi_{k, 1}$ are mixed separately with the rotating bin, then $\pi_{k, 0}$ and $\pi_{k, 1}$ are each mixed separately with the product of both of these mixings, etc. After each mixing with a permutation bin, the renormalization bin is mixed with the product. After all permutation sequences are created, the fixed bin is mixed with each, creating single assemblies with label strings $P_{k, i}$ for all $0 \leq i<k$.

Combining Interleaved Assemblies The final step is to combine each assembly with label $P_{k, i}$ into a single assembly. Each assembly is contained in a separate bin after its production, and has glue 0 on its west side and glue $2 k$ on its east side. To the assembly with label $P_{k, i}$, the tiles $(2 k+1+i)[1] 0$ and $(2 k)[0](2 k+2+i)$ are added. Then these assemblies are combined to produce a single long assembly with glue $(2 k+1)$ on the west side, and glue $(3 k+1)$ on the east. To finish off the assembly, two more tiles are added: $(n u l l)[0](2 k+1)$ and $(3 k+1)[1]($ null $)$. This ensures that the final result is an assembly with the label $S_{k}$ and null glues on both ends.

Theorem 3. The SAS described in Section 5.2 has size $O(k)$.

Proof. Break the SAS into the following sections:

1. Creating the $a$-bin and $b$-bin. 
2. Creating the permutation and renormalization bins.

3. Creating the interleaved assemblies.

4. Combining interleave assemblies.

Item 1 requires $O(k)$ edges to create a tile for each symbol in $A_{k}$ or $B_{k}$ respectively and mixing them together. Item 2 requires $O(k)$ edges to create three bins each with a pair of tiles for each $c$-buffered element of the $b$-bin. Item 3 requires $O(k)$ edges: this portion of the mix DAG resembles an upsidedown tree and contains no more than two leaves per permutation assembly. Item 4 requires $O(1)$ edges per assembly (and thus $O(k)$ edges total) to add two location-specifying tiles and combine it with the other assemblies into a single bin. In total $k$ interleave assemblies (one per shift) are created, so $O(k)$ edges are in this portion of the mix DAG. Combining interleave assemblies is done by adding at most two tiles to each interleave assembly followed by combining them into a single bin. A constant number of edges exist for each assembly, so $O(k)$ edges exist in this portion of the mix DAG.

\subsection{An RCFG Lower Bound for $S_{k}$}

The following definition and theorem are taken from [7].

Definition 12. As defined in [3], the size of the LZ-factorization of a string $s$ (denoted $|L Z(s)|$ ) is the number of elements generated by the LZ77 algorithm without self-referencing.

Theorem 4. For an RCFG G generating a string $s,|L Z(s)| \leq|G|$.

Lemma 2. All factors in the LZ-factorization of $S_{k}$ have size $<16\lceil\log k\rceil+26$.

Proof. Assume, for the sake of contradiction, the LZ-factorization of $S_{k}$ contains some factor $y$ of size $\geq 16\lceil\log k\rceil+26$. Then the factor is long enough that there must be some $i, j$ such that $C_{k, i, j}$ is a substring of $y$. Let $x$ be the part of the string preceding $y$. Then by the definition of LZ factorization, $y$ is a substring of $x$, and therefore $C_{k, i, j}$ is a substring of $x$.

$C_{k, i, j}$ contains as a substring the string $A_{k, j}$. To ensure the correct parity on runs of characters, the portion of $x$ where $A_{k, j}$ is found must have been completely generated by some other $A_{k, j^{*}}$. Then it must be that $A_{k, j}=A_{k, j^{*}}$, and by Definition 9 it follows that $j=j^{*}$. So the portion of $x$ where $C_{k, i, j}$ is found must have been completely generated by some other $C_{k, i^{*}, j}$, where $i \neq i^{*}$. Then $A_{k, k+\Pi_{k,\lceil\log k\rceil, i}(j)}=A_{k, k+\Pi_{k,\lceil\log k\rceil, i^{*}(j)}}$. By Definition 9 it follows that $k+\Pi_{k,[\log k\rceil, i}(j)=k+\Pi_{k,\lceil\log k\rceil, i^{*}}(j)$. Therefore, by Definition[10, $i=i^{*}$, which gives us a contradiction.

Theorem 5. The smallest $C F G$ that can be used to construct $S_{k}$ has size $\Omega\left(k^{2}\right)$.

Proof. By Lemma 2, the maximum length of an LZ factor is $16\lceil\log k\rceil+29$. The sum of the lengths of the LZ factors is equal to $\left|S_{k}\right|=\Theta\left(k^{2} \log k\right)$. Hence, the number of LZ factors is $\Omega\left(k^{2}\right)$. By Theorem 4 , the size of the minimum grammar must therefore be $\Omega\left(k^{2}\right)$. 


\subsection{Asymptotic Separation of SASs and RCFGs for $S_{k}$}

Separation refers to the minimum difference in size between an RCFG and a SAS generating the same (label) string. Here we show the separation achieved for the strings $S_{k}$, where $k$ is the number of glues used to generate the label string $S_{k}$ by the SAS in Section 5.3 and $n$ is the length of $S_{k}$.

Corollary 2. The strings $S_{k}$ have separation $\Omega(k)$.

Proof. By Theorem 5, any RCFG generating $S_{k}$ has size $\Omega\left(k^{2}\right)$. By Theorem 3 . a self-assembly system of size $O(k)$ exists that produces an assembly with label string $S_{k}$. So the ratio of the size of any grammar generating $S_{k}$ to the size of some SAS instance is $\Omega(k)$.

Corollary 3. The strings $S_{k}$ have separation $\Omega(\sqrt{n / \log n})$.

Proof. The length of $S_{k}$ is $\Theta\left(k^{2} \log k\right)$. So $k=\Theta(\sqrt{n / \log n})$. By Corollary 2, the separation is $\Omega(k)$. So the separation is also $\Omega(\sqrt{n / \log n})$.

Given that the number of glues is limited in practice, it is natural to consider whether $\Omega(k)$ separation is possible for $k$ glues where $k \ll n$. We show this is possible for $k=\Theta(\log n)$.

Definition 13. Define the recursive string $T_{k, t}=01 \circ T_{k, t-1} \circ 01 \circ T_{k, t-1} \circ 01$, where $T_{k, 1}=S_{k}$. The length of $T_{k, t}$ is $\Theta\left(2^{t}\left|S_{k}\right|\right)=\Theta\left(2^{t} k^{2} \log k\right)$.

Theorem 6. The strings $T_{k, k}$ have separation $\Omega(k)$ and use $\Theta(\log n)$ glues.

Proof. Since $T_{k, k}$ has $S_{k}$ as a substring, any CFG generating $T_{k, k}$ has size $\Omega\left(k^{2}\right)$ by Theorem 5. To construct a SAS to generate this string, we first use the SAS described in Section 5.2 to generate an assembly $a\left[S_{k}\right] b$. We can then add a constant number of tiles to get two assemblies $c\left[1 S_{k} 0\right] e$ and $e\left[1 S_{k} 0\right] d$, which when combined create the assembly $c\left[1 S_{k} 01 S_{k} 0\right] d$. We then add two more tiles to construct the assembly $a\left[01 S_{k} 01 S_{k} 01\right] b$. This process can then be repeated $k$ times. In total $O(k)$ additional work is performed, so the new SAS has size $O(k)$. The length $n$ of the string is $\Theta\left(2^{k} k^{2} \log k\right)$, so $k=\Theta(\log n)$.

\subsection{Upper Bounds for Separation of SASs and RCFGs}

The PSASs described in Section 5.2 constructing $S_{k}$ used $O(k)$ distinct glue pairs to achieve a separation of $\Omega(k)$. We now show bounds on the worst-case separation in terms of the number of glues $k$ and the length of the string $n$.

Lemma 3. Given a minimal $S A S A$, any two distinct assemblies $A_{1}$ and $A_{2}$ in the same bin must have different glues on either the west side or the east side. 
Proof. For the sake of contradiction, say that there is a distinct pair of assemblies $A_{1}$ and $A_{2}$ with matching glues on both the west and east sides of the assemblies. Because the accessible glues on both assemblies are identical, any assembly which adheres to $A_{1}$ must also adhere to $A_{2}$, and vice versa. Hence, for every superassembly of $A_{1}$, there is a corresponding superassembly of $A_{2}$ in the same bin with the same accessible glues, but a different label sequence. Any attempt to merge two such assemblies to create a single assembly results in an infinite label sequence. So the SAS $A$ cannot produce a single goal assembly, violating the definition of a SAS.

Corollary 4. Given a minimal SAS A using $k$ glues to produce a string s, each bin in $A$ contains at most $k^{2}$ distinct assemblies.

Lemma 4. Given a $S A S A$ using $k$ glues and generating an assembly with label string s, an $R C F G$ of size $O\left(k^{2}|A|\right)$ generating $s$ can be constructed.

Proof. For each bin in $A$ and each distinct assembly in that bin, construct one bin in the SSAS $B$. By Corollary 4 , the number of bins in $B$ will be at most $k^{2}$ times the number of bins in $A$.

Now consider what happens when $\ell$ bins in $A$ are simultaneously mixed to produce a single bin $c$ containing several assemblies. How many edges must we add to $B$ to ensure that each assembly in $c$ is correctly constructed in $B$ ? To determine this, we define $G$ to be a directed graph with a node corresponding to each glue and, for each distinct input assembly $g_{1}[s] g_{2}$, a directed edge from $g_{1}$ to $g_{2}$. Then each distinct assembly in $c$ corresponds to a source-sink pair in $G$, and each possible way to construct that assembly corresponds to a path in $G$ from the source of the assembly to the sink of the assembly.

Say that there exist three glues $g_{1}, g_{2}, g_{3}$ such that $\left(g_{1}, g_{2}\right)$ and $\left(g_{2}, g_{3}\right)$ are edges in $G$ but $\left(g_{1}, g_{3}\right)$ is not an edge in $G$. Then we can mix the assembly corresponding to the edge $\left(g_{1}, g_{2}\right)$ with the assembly corresponding to the edge $\left(g_{2}, g_{3}\right)$ to get an assembly with glue $g_{1}$ to the west and glue $g_{3}$ to the east. This is equivalent to adding the edge $\left(g_{1}, g_{3}\right)$ to $G$. Each such mixing requires us to add a constant number of nodes and edges to the mix DAG $B$, and increases the number of edges in $G$ by 1 . The graph $G$ can never have more than $k^{2}$ edges, so repeated mixings of this type add a total of $O\left(k^{2}\right)$ work to $B$. Hence, any mixing of bins in $A$ can be replaced by $O\left(k^{2}\right)$ binary mixes in $B$. As a result, $|B|$ has size $O\left(k^{2}|A|\right)$, and can therefore be converted to an RCFG with size $O\left(k^{2}|A|\right)$ by Theorem 2 .

Theorem 7. With respect to the total number of distinct glue pairs $k$, the separation for any string is $O\left(k^{2}\right)$.

Proof. Let $A$ be a SAS using $k$ glues that generates a string $s$. By Lemma 4 , there is an RCFG of size $O\left(k^{2}|A|\right)$ that generates $s$. So separation is at most $O\left(k^{2}\right)$.

Theorem 8. With respect to the length of the string $n$, the separation for any binary string is $O\left((n / \log n)^{2 / 3}\right)$. 
Proof. Let $A$ be a SAS generating a string $s$ of length $n$. Let $k$ be the number of glues used in $A$. Either $k=O\left((n / \log n)^{1 / 3}\right)$ or $k=\omega\left((n / \log n)^{1 / 3}\right)$. If $k=O\left((n / \log n)^{1 / 3}\right)$ then by Lemma 4 there is an RCFG of size $O\left(k^{2}|A|\right)=$ $O\left((n / \log n)^{2 / 3} \cdot|A|\right)$ generating $s$. So the separation is at most $O\left((n / \log n)^{2 / 3}\right)$. Now suppose $k$ is $\omega\left((n / \log n)^{1 / 3}\right)$. Then $|A|=\omega\left((n / \log n)^{1 / 3}\right)$. Lemma 2 of Section 2.2 in [5] shows that there is an RCFG of size $O(n / \log n)$ generating $s$. Hence, the separation is $o\left((n / \log n)^{2 / 3}\right)$. So in both cases the separation is $O\left((n / \log n)^{2 / 3}\right)$.

\section{Acknowledgments}

We thank Martin Demaine, André Schulz, Diane Souvaine, and Hyunmin Yi for helpful discussions, and anonymous reviewers for helpful suggestions.

\section{References}

1. Moses Charikar, Eric Lehman, Ding Liu, Rina Panigrahy, Manoj Prabhakaran, April Rasala, Amit Sahai, and abhi shelat. Approximating the smallest grammar: Kolmogorov complexity in natural models. In Proceedings of the 34th Annual ACM Symposium on Theory of Computing, pages 792-801, New York, NY, USA, 2002. ACM.

2. Erik Demaine, Martin Demaine, Sndor Fekete, Mashhood Ishaque, Eynat Rafalin, Robert Schweller, and Diane Souvaine. Staged self-assembly: nanomanufacture of arbitrary shapes with o(1) glues. Natural Computing, 7:347-370, 2008.

3. Martin Farach and Mikkel Thorup. String matching in Lempel-Ziv compressed strings. Algorithmica, 20:388-404, 1998.

4. Mika Göös and Pekka Orponen. Synthesizing minimal tile sets for patterned dna self-assembly. In Yasubumi Sakakibara and Yongli Mi, editors, DNA Computing and Molecular Programming, volume 6518 of Lecture Notes in Computer Science, pages 71-82. Springer Berlin / Heidelberg, 2011.

5. Eric Lehman. Approximation Algorithms for Grammar-Based Data Compression. PhD thesis, MIT, 2002.

6. Xiaojun Ma and Fabrizio Lombardi. Synthesis of tile sets for dna self-assembly. Computer-Aided Design of Integrated Circuits and Systems, IEEE Transactions on, 27(5):963-967, may 2008.

7. Wojciech Rytter. Application of Lempel-Ziv factorization to the approximation of grammar-based compression. In Alberto Apostolico and Masayuki Takeda, editors, Combinatorial Pattern Matching, volume 2373 of Lecture Notes in Computer Science, pages 20-31. Springer Berlin / Heidelberg, 2002. 10.1007/3-540-45452-7_3.

8. Hiroshi Sakamoto. A fully linear-time approximation algorithm for grammar-based compression. Journal of Discrete Algorithms, 3(2-4):416-430, 2005.

9. David Soloveichik and Erik Winfree. Complexity of self-assembled shapes. In DNA Computing, volume 3384 of Lecture Notes in Computer Science, pages 344-354. 2005.

10. Erik Winfree. Algorithmic Self-Assembly of DNA. PhD thesis, Caltech, 1998. 